\title{
NUMBER THEORETICAL DEVELOPMENTS ARISING FROM THE SIEGEL FORMULA
}

\author{
BY S. J. HARIS
}

1. Introduction. Siegel Formula is the name Weil gave to an equality which relates a theta series with an Eisenstein series [16]. The original result of Siegel is quite arithmetic in nature, with special cases yielding for example Fermat's theorem that every prime $p \equiv 1(\bmod 4)$ is expressible as a sum of two squares in essentially one way; Jacobi's theorem on the number of ways of writing an integer as a sum of four squares-namely $8 \Sigma_{2 d+1 \mid n}(2 d+1)$ for $n$ odd or $24 \sum_{2 d+1 \mid n}(2 d+1)$ for $n$ even; and also Dirichlet's class number formula. In the more arithmetically accessible case, Siegel's theorem can be stated as follows:

Let $h$ be a positive definite quadratic form in $m$ variables, with integer coefficients, write

$$
\begin{aligned}
d(t) & =\#\left\{x \in \mathbf{Z}^{m} \mid h(x)=t\right\}, \\
d_{p}(t) & =\operatorname{limit}_{t \rightarrow \infty} \frac{\#\left\{x \in\left(\mathbf{Z} / p^{r} \mathbf{Z}\right)^{m} \mid h(x) \equiv t\left(\bmod p^{r}\right)\right\}}{p^{r(m-1)}}, \\
d_{\infty}(t) & =\operatorname{limit}_{D \rightarrow\{t\}} \frac{\text { volume } h^{-1}(D)}{\text { volume }(D)},
\end{aligned}
$$

where $D$ runs through the compact neighbourhoods of $t$ and $h: \mathbf{R}^{m} \rightarrow \mathbf{R}$. Then we have $d(t)=d_{\infty}(t) \Pi_{p} d_{p}(t)$. (At least this formulation is correct when $m>2$ and the genus of $h$ has only one class. See $\S 6$ for a discussion of genus, class of $h$.)

In general Siegel characterises his result as having the same quantitative relationship to the Hasse-Minkowski theorem, as the Jacobi Theorem mentioned above has to Lagrange's result that every integer is a sum of four squares.

One can ask also for the number of representations of an $n \times n$ integral, symmetric, positive definite matrix $T$ by a given $m \times m$ one $S$, and once more Siegel has a similar result. Moreover, Siegel has generalized his theorem to $T$ indefinite and where the coefficients lie in an algebraic number field only now the definition of the densities $d_{p}(t)$ are much more involved, with no ready arithmetic interpretation. The proofs consist of constructing an Eisenstein series $E(\tau)$ which behaves like the generating function $f(\tau)$ for our Diophantine problem:

Received by the editors March 15, 1979.

AMS (MOS) subject classifications (1970). Primary 10-02, 14 G99.

Key words and phrases. Poisson summation formula, asymptotic expansions and Mellin transforms, Riemann hypothesis for hypersurfaces. 
if $A(T, S)=$ the number of representations of $T$ by $S$,

$$
\begin{aligned}
f(\tau) & =\sum_{\xi \in M_{m, n}(\mathrm{Z})} e^{\pi i \operatorname{trace}\left({ }^{\prime} \xi S \xi \tau\right)} \\
& =\sum_{T \in M_{m, n}(\mathbf{Z})} A(T, S) e^{\pi i \operatorname{trace}(T \tau)}
\end{aligned}
$$

(where $\tau$ is in the Siegel upper half space), then $E(\tau)$ is to behave like $f(\tau)$ under all modular substitutions. One recovers the " $d(t)=d_{\infty}(t) \Pi_{p} d_{p}(t)$ " formulation from these analytic functions by equating Fourier coefficients [13, p. 180].

Tamagawa [14] observed that one can view the $d_{p}(t)$ 's also as limits of measures, in a $p$-adic sense, enabling Weil to recast Siegel's theorem in the language of adelic analysis and distributions-and to examine the role of the symplectic group in these results. We shall discuss Weil's framework in $\$ 5$ and show that by a suitable choice of Schwartz-Bruhat function one can recover Siegel's theorem on the genus invariant (\$6). All these considerations are for quadratic forms.

In another direction, pursuing the Hardy-Littlewood circle method, Davenport obtained a result on cubic forms [3], which Birch [1] extended to higher degree forms, by the use of some algebraic-geometric techniques. The Davenport-Birch theorem asserts that for $f$, a homogeneous form of degree $d$, with rational coefficients, the variety $f=0$ has a rational point if it has a nonsingular real point and a nonsingular $p$-adic point for every $p$, provided that the singular locus of $f=0$ has codimension sufficiently large compared with the degree $d$.

The developments referred to in the title are the recent results of Igusa, which, among other things, yield a Hasse-Minkowski type result as the Davenport-Birch theorem for certain $f$, but from the Weil formulation. The spirit of approach is the same as Weil's, with the Poisson Formula playing a key role, but for forms of degree higher than two. The strength of this approach is that when ultimately all of Weil's techniques have been generalized and in particular a suitable "metaplectic group" found, more will follow, just as the Hasse-Minkowski theorem for quadratic forms is a purely qualitative consequence of the Siegel Formula, but which has numerous quantitative consequences as well (for example: the Tamagawa numbers of many of the classical groups follow from the Siegel Formula of Weil). Igusa's work is a beautiful blend of algebraic geometry and analysis to yield arithmetic results, as we will show.

In $\$ 2$ we outline Weil's abstract Poisson Formula. Igusa proves that for certain $f$ this holds, if estimates of some associated transforms are satisfied (Theorem 2), provided that the singularities of $f=0$ are at least of dimension 2 less than the dimension of the hypersurface. In $\$ 4$ we will indicate how for nonsingular $f=0$, these estimates are satisfied-by an application of the Riemann hypothesis for such hypersurfaces. Further, we will indicate how the recognizably arithmetic result (the Hasse-Minkowski theorem for $f=0$ ) follows from the harmonic analysis and algebraic geometry. The fact that some restrictions are necessary on $f$ is not surprising, since for example, for 
the hypersurface: $3\left(x_{1}^{2}+\cdots+x_{n-2}^{2}\right)^{3}-4 x_{n-1}^{6}-5 x_{n}^{6}=0$ we have nonsingular real points, as well as nonsingular $p$-adic points for every $p$, yet it has no rational zero [11]. But here the theory is not applicable since the locus of singular points: $x_{1}^{2}+\cdots+x_{n-2}^{2}=x_{n-1}=x_{n}=0$ is too large.

Finally in $\S 7$ we mention further applications of Igusa's theory.

We take this opportunity to thank Professor Igusa for introducing us to this branch of number theory, through his lectures at Johns Hopkins on Weil's two paper [15], [16].

Before embarking, let us recall some notations and concepts.

A locally compact abelian group $X$ is the limit of elementary pairs of subgroups $\left(H, H^{\prime}\right)$ in the following sense: we can find an arbitrarily large open subgroup $H \subset X$, which contains an arbitrarily small compact subgroup $H^{\prime} \subset H$ such that the quotient group $H / H^{\prime}$ is isomorphic to a direct product of a finite group and finitely many copies of $\mathbf{R}, \mathbf{Z}$ and $\mathbf{T}$ (here $\mathbf{R}$ is the real number, $\mathbf{Z}$ the integers and $\mathbf{T}=\mathbf{R} / \mathbf{Z}$, the unit circle).

For $X$ a locally compact abelian group, the space $\delta(X)$ of Schwartz-Bruhat functions is defined as follows: for $E$ elementary, $\Phi: E \rightarrow \mathbf{C}$ is to belong to $\delta(E)$ if $\|p(x) D \Phi(x)\|$ is finite for each polynomial $p(x)$ and each invariant differential operator $D$ on $E$, where $\|-\|$ is the sup norm. Then $\|p D \Phi\|$ is a seminorm on $\delta(E)$ and the collection of all of these defines the topology on $\delta(E)$. Now if $\left(H, H^{\prime}\right)$ is an elementary pair, set $\delta\left(H, H^{\prime}\right)=$ the complex valued functions on $X$ whose support is in $H$, which are constant on the cosets of $H^{\prime}$ and whose projection to $H / H^{\prime}$ belongs to $\delta\left(H / H^{\prime}\right) . \delta(X)$ is then the inductive limit, over all elementary pairs $\left(H, H^{\prime}\right)$ of the topologial vector spaces $\delta\left(H, H^{\prime}\right)$. These are the functions we will be concerned with, for special $X$.

Another ingredient will be adelisation. Chevalley introduced adeles (in 1936) in connection with an algebraic approach to class field theory, while Tate (1950) used "valuation vectors." These are two instances of "adelisation," a language that is ideally suited to treating questions concerned with the interplay between local and global properties of varieties. Given a variety $X$ defined over a global field $k$ (that is either a number field or a function field of dimension one over a finite field) we can associate with $X$ a locally compact topological space $X_{A}$, as follows: for every valuation $v$ of $k$, let $X_{v}$ denote the $k_{v}$-rational points of $X$. These spaces $X_{v}$ are locally compact for every $v$, but unfortunately the easy way of putting these together, namely $\Pi_{v} X_{v}$, is not locally compact, so we must be more clever. Let $X_{v}^{0}$ denote the $\mathfrak{D}_{v}$-rational points of $X$, where for $v$ nonarchimedean, $\mathfrak{S}_{v}$ is the ring of integers in $k_{v}$, so the $X_{v}^{0}$ are compact. Now if $S$ is a finite set of valuations of $k$, containing all the archimedean ones, we put $X_{S}=\Pi_{v \in S} X_{v} \times \Pi_{v \notin S} X_{v}^{0}$, which, with the product topology, is locally compact. Then we take $X_{A}=$ $\cup_{S} X_{S}$, the union being over all such sets $S$, with topology: 0 open in $X_{A}$ iff $0 \cap X_{S}$ is open in $X_{S}$, for all $S . X_{A}$ is a locally compact space.

For example, for $X=G_{a}=\left\{\left(\begin{array}{c}1 x \\ 01\end{array}\right) \mid x \in \Omega\right\}$, the additive group, $X_{A}=k_{A}$, the group of adeles of $k$. In particular $Q_{A}=\left\{\left(x_{v}\right) \in \Pi Q_{v} \mid x_{p} \in \mathbf{Z}_{p}\right.$ for almost all $p$. Note that $Q \subset Q_{A}$, via the diagonal embedding and it is a discrete subgroup. Amazingly, the quotient $Q_{A} / Q$ is connected, amazing when we consider that for all but one factor $Q_{v}=\mathbf{R}, Q_{A}$ is built up from totally 
disconnected spaces. If $X$ is a finite-dimensional vector space over $k$, then $X_{A}$ is a locally compact abelian group.

For certain varieties $X$, we can define a positive measure on the space $X_{A}$. Let $\omega$ be a differential form of degree $=$ dimension $X$ and suppose $\omega$ never vanishes on $X$. We can define a measure $|\omega|_{v}$ on $X_{v}$, then multiply them together to obtain $|\omega|_{A}$ on $X_{A}$. Often one needs "convergence factors" to ensure that the resulting produce measure converges. It is a fact that for the varieties we shall consider, we may take these convergence factors to be 1 [17]. The measure $|\omega|_{v}$ is given by: $\omega=f(x) d x_{1} \wedge \cdots \wedge d x_{n}$ locally, and set $|\omega|_{v}=|f(x)|_{v}\left|d x_{1}\right|_{v} \cdots\left|d x_{n}\right|_{v}$, where for $a \neq 0 \in k_{v}|a|_{v}$ is the module of the automorphism $x \rightarrow a x$, while $|0|_{v}=0$. Then we obtain $|\omega|_{v}$ on $X_{v}$ by patching together the local charts.

2. Abstract Poisson Formula [16]. Let $X \stackrel{f}{\rightarrow} G$ be a continuous mapping of locally compact abelian groups and fix Haar measures $d x, d g$ on $X, G$. Let $G^{*}$ denote the Pontryagin dual of $G$ and for $\Phi \in \mathcal{S}(X)$, set

$$
F_{\Phi}^{*}\left(g^{*}\right)=\int_{X} \Phi(X)<f(x), \quad g^{*}>d x .
$$

This defines a continuous, bounded function on $G^{*}$ for every $\Phi$. We are interested in decomposing the measure $d x$ into measures carried along the fibres $f^{-1}(g)$.

WeIL's CONDITION (A). If the function $F_{\Phi}^{*}$ is integrable on $G^{*}$ and if $\int_{G^{*}}\left|F_{\Phi}^{*}\left(g^{*}\right)\right| d g^{*}$ is uniformly convergent on all compact subsets of $\delta(X)$, then there exists a unique family of positive measures $d \mu_{g}$ on $X$, with support $d \mu_{g} \subset f^{-1}(g)$, such that for every $\Phi \in L(X)$ (= continuous functions of compact support), the function $F_{\Phi}(g)=\int_{X} \Phi(x) d \mu_{g}(x)$ is continuous on $G$ and satisfies $\int_{G} F_{\Phi} d g=\int_{X} \Phi d x-(*)$. In fact, the $d \mu_{g}$ are tempered measures and for all $\Phi \in \mathcal{S}(X)$, the $F_{\Phi}$ are continuous, belong to $L^{1}(G)$, satisfy (*) and have as their Fourier transform the $F_{\Phi}^{*}$.

This theorem ensures the existence of the decomposition of the measure $d x$. The next result guarantees that the conditions of (A) are met, as well as the Poisson Formula. Let $\Gamma$ be a discrete subgroup of $G$ such that $G / \Gamma$ is compact and let $\Gamma_{*}$ denote the annihilator of $\Gamma$ in $G^{*}$.

Condition (B). Suppose that for all $\Phi \in \mathcal{S}(X), g^{*} \in G^{*}$, the series $\Sigma_{\gamma^{*} \in \Gamma_{*}}\left|F_{\Phi}^{*}\left(g^{*}+\gamma^{*}\right)\right|$ is convergent and uniformly so on all compact subsets of $\mathcal{S}(X) \times G^{*}$; then condition (A) is satisfied for the mapping $X \stackrel{f}{\rightarrow} G$ and we have the Poisson Formula:

$$
\sum_{\gamma \in \Gamma} F_{\Phi}(\gamma)=\sum_{\gamma^{*} \in \Gamma_{*}} F_{\Phi}^{*}\left(\gamma^{*}\right)
$$

Notice that if $F_{\Phi}, F_{\Phi}^{*}$ would be Schwartz-Bruhat functions, this latter equality would be trivial, but in general the $F_{\Phi}, F_{\Phi}^{*}$ are far from being such functions. The above two theorems follow from functional analysis-generalities on Fourier inversion, Fubini's theorem and tempered distributions. They occupy a short part of Weil's paper. The number theory enters when one makes the measures $d \mu_{g}$ explicit and in verifying condition (B), as we will outline in $\S 5$. 
Now condition (B) has two parts. Writing $\Phi_{g^{*}}(x)=\Phi(x)\left\langle f(x), g^{*}\right\rangle$, (B) follows from:

$\left(\mathrm{B}_{0}\right)$ the mapping

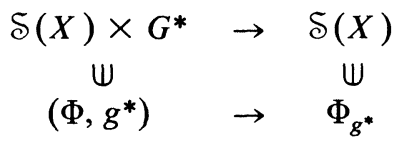

is continuous,

$\left(\mathrm{B}_{1}\right)$ the series $\sum_{\gamma^{*} \in \Gamma_{*}}\left|F_{\Phi}^{*}\left(\gamma^{*}\right)\right|$ is uniformly convergent on compact subsets of $\mathcal{S}(X)$.

Whenever $f$ is defined by polynomials, condition $\left(B_{0}\right)$ is satisfied [10], so one must verify $\left(\mathrm{B}_{1}\right)$. For quadratic $f$, this follows from reduction theory applied to the metaplectic group; for higher degree forms, it is one of the main points of Igusa's work.

3. The Poisson Formula for the higher degree forms. We now come to the main topic of this article. For $k$ any field, a polynomial $f(x) \in k\left[x_{1}, \ldots, x_{n}\right]$ gives rise to the mapping $f: X=A^{n} \rightarrow A$ of affine $n$-space, all defind over $k$. Let

$$
S_{f}=\left\{x \in X \mid \partial f / \partial x_{1}=\cdots=\partial f / \partial x_{n}=0\right\},
$$

the critical set of $f$. Note that by Euler's result, if $\operatorname{char}(k) \nmid$ degree $f, f$ homogeneous, then $S_{f} \subset f^{-1}(0)$. In general we say $f$ is "almost homogeneous" when $S_{f} \subset f^{-1}(0)$. In explaining the results, Weil's language of algebraic geometry suffices, although as it will become apparent shortly, the proofs rely heavily on all of the Grothendieck machinery. So, let $\Omega$ be a universal domain containing $k$, and for $i \in \Omega$, set $U(i)=f^{-1}(i)-S_{f}$, a nonsingular variety. Write $d x=d x_{1} \wedge \cdots \wedge d x_{n}$ for the $n$-form on $X$. Then we can express it as $d x=d f \wedge \theta$ for an $(n-1)$-form $\theta$ on $X$. In fact we can choose $\theta$ so that it is regular along $U(i)$, when $\theta_{i}=\left.\theta\right|_{U(i)}$ is well defined.

Since the explicit determination of the measures $d \mu_{g}$ involves these $\theta_{i}$, let us consider an example. Let $f(x, y)=x^{3} y+x^{2} y^{2}$. Then $S_{f}=\{(0, y) \mid y$ arbitrary $\}$ and we need $\theta=A d x+B d y$ such that $A\left(x^{3}+2 x^{2} y\right)-B\left(3 x^{2} y+2 x y^{2}\right)=$ 1. Now if $i \neq 0,(x, y) \in U(i) \Leftrightarrow x^{3} y+x^{2} y^{2}=i \neq 0$, so in particular $x y \neq 0$ and we may take

$$
A=-9 / 8 x y^{2}, \quad B=-(3 x+4 y) / 8 x y^{3}
$$

while $(x, y) \in U(0) \Leftrightarrow x^{3} y+x^{2} y^{2}=0, x \neq 0$, so that we may take $A=(1-$ $y) /\left(x^{3}+2 x^{2} y\right), B=1 /\left(3 x^{2}+2 x y\right)$. The reader familiar with complex analysis will recognize that the $\theta_{i}$ 's are just the residues of $(f(x)-i)^{-1} d x$ along the fibers over $i$.

From here on, we assume that $k$ is a global field, with $k_{v}$ the completions of $k$ and $k_{A}$ the adele ring of $k$. Fix a nontrivial character $\psi$ of $k_{A} / k$. We further assume that $f$ is almost homogeneous. Then $X$ is an $n$-dimensional vector space defined over $k$ and put $X_{v}$ (respectively $X_{A}$ ) into Pontryagin duality with itself by $\psi_{v}([x, y])(\operatorname{resp} . \psi([x, y]))$ where $[x, y]=\Sigma_{i} x_{i} y_{i}$. 
(1) LOCAL CASE. We can explicitly describe the Schwartz-Bruhat functions in this case:

$$
\delta\left(X_{v}\right)=\left\{\begin{array}{l}
\text { rapidly decreasing } C^{\infty} \text {-functions on } X_{v}, \text { if } v \in S_{\infty}, \\
\text { locally constant functions of compact support, if } v \notin S_{\infty} .
\end{array}\right.
$$

The assumption that $f$ is almost homogeneous means that $U(i)_{v}=f(i)_{v}$ for $i \neq 0 \in k_{v}$ and define

$$
F_{\Phi_{v}}(i)=\int_{U(i)_{v}} \Phi_{v}\left|\theta_{i}\right|_{v} \quad \text { for } i \in k_{v}^{x}
$$

Here $\left|\theta_{i}\right|_{v}$ is the measure that the residue $\theta_{i}$ defines on $U(i)_{v}$, as we outlined earlier. Easy estimates and Fubini's theorem show that $F_{\Phi_{i}}(i)$ is a continuous function on $k_{v}^{x}$ for all $\Phi_{v} \in \mathcal{S}\left(X_{v}\right)$ and regarding it as an $L^{1}$ function on $k_{v}$ it has $F_{\Phi_{v}}^{*}\left(i^{*}\right)=\int_{X_{v}} \Phi_{v}(x) \psi_{v}\left(i^{*} f(x)\right)|d x|_{v}$ as its Fourier transform. Further the equality $\int_{k_{v}} F_{\Phi_{v}}(i)|d i|_{v}=\int_{X} \Phi_{v}(x)|d x|_{v}$ holds. The point is that for $i \neq 0$ in the local case, the measures $\left|\theta_{i}\right|_{v}$ work as the " $d \mu_{g}$ " that would follow from the condition (A), with only the mild assumption that $f$ be almost homogeneous. The conclusion of condition (A) is that the functions $F_{\Phi}(g)$ are continuous on $G$, so we are faced with the question: does $\operatorname{limit}_{i \rightarrow 0} F_{\Phi_{v}}(i)$ exist? Denote the limit $\boldsymbol{F}_{\Phi_{v}}(0)$ when it exists, Igusa's first contribution is an analysis of this question and it involves some deep algebraic geometry, in the form of a Hironaka resolution of the singularities of $f=0$. In its simplest form, this consists in "blowing up a point on a variety". More generally it is a finite sequence of monoidal transformations. Briefly, the situation is this in $\mathbf{C}^{2}$. If $\mathbf{P}^{1}$ denotes the projective line and $[\xi, \eta]$ the point in $\mathbf{P}^{1}$ defined by $(\xi, \eta) \in \mathbf{C}^{2}$ - $(0,0)$, consider $\mathbf{C}^{2} \times \mathbf{P}^{1} \supset\left(\mathbf{C}^{2}-(0,0)\right) \times \mathbf{P}^{1} \supset W=\{((x, y),[\xi, \eta]) \mid[x, y]$ $=[\xi, \eta]\}$. Let $\bar{W}$ be the closure of $W$ in $\mathbf{C}^{2} \times \mathbf{P}^{1}$; then $\bar{W}=\{((x, y),[\xi, \eta]) \mid x \eta$ $=y \xi\} \cdot \bar{W}$ is a manifold with a projection $\pi: \bar{W} \rightarrow \mathbf{C}^{2}$. For $p \in \mathbf{C}^{2}$,

$$
\pi^{-1}(p)=\left\{\begin{array}{l}
\text { single point if } p \neq(0,0) \\
\mathbf{P}^{1} \text { if } p=(0,0)
\end{array}\right.
$$

Then $\bar{W}$ is called the monoidal transformation centered at $(0,0)$ of $\mathbf{C}^{2}$ and denote it by $\mu_{0}\left(\mathbf{C}^{2}\right)$ say. Similarly for $\mu_{0}\left(\mathbf{C}^{n}\right)$. Now for a manifold $M$ of dimension $n$, with a submanifold $N \subset M$ of dimension $k$, if $n-k \geqslant 2$ we have $\mu_{N}(M)$ defined as follows: locally $M \approx \mathbf{C}^{n-k} \times \mathbf{C}^{k}$ where the second factor is $N$ locally while the first factor is the normal to $N$ locally. Set $\mu_{\mathbf{C}^{k}}\left(\mathbf{C}^{n-k} \times \mathbf{C}^{k}\right)=\mu_{0}\left(\mathbf{C}^{n-k}\right) \times \mathbf{C}^{k}$. Then these patch together to form $\mu_{N}(M)$. Hironaka's theorem asserts that if $\operatorname{chr}(k)=0$, the algebraic geometric analogue always exists, which by a finite sequence of monoidal transformations yields a smooth variety in birational correspondence with the variety $f=0$.

For our purposes, we assume that such a resolution exists for $f=0$.

THEOREM 1 (IguSA) [5], [7]. (i) $F_{\Phi_{v}}(0)$ exists if and only if $F_{\Phi_{v}}^{*} \in L^{1}\left(k_{v}\right)$;

(ii) $F_{\Phi_{v}}^{*} \in L^{\prime}\left(k_{v}\right)$ if and only if $\left|F_{\Phi_{v}}^{*}\left(i^{*}\right)\right| \leqslant$ const $\max \left(1,\left|i^{*}\right|_{v}\right)^{-\sigma}$ for a fixed $\sigma>1$, for every $i^{*} \in k_{v}$. Here the constant depends on $\Phi_{v}$.

Suppose the condition (ii) is satisfied for every $\Phi_{v} \in \mathcal{S}\left(X_{v}\right)$. Then the mapping “ $\Phi_{v} \rightarrow F_{\Phi_{v}}(0)-\int_{U(0)_{v}} \Phi_{v}\left|\theta_{0}\right|_{v}$ " defines a positive tempered measure on $X_{v}$, with support contained in $S_{v}=f^{-1}(0)_{v}-U(0)_{v}$. Then 
(iii) the equality $F_{\Phi_{v}}(0)=\int_{U(0)_{v}} \Phi_{v}\left|\theta_{0}\right|_{v}$ for one $\Phi \geqslant 0$, smooth, of compact support implies a certain geometric property $(\mathrm{P})$ for the Hironaka resolution of $f=0$. Conversely, if property $(\mathrm{P})$ holds, then $F_{\Phi_{v}}(0)=\int_{U(0)_{v}} \Phi_{v} \mid \theta_{0} l_{v}$ holds for every $\Phi_{v} \in \mathcal{S}\left(X_{v}\right)$.

The proof of this theorem is contained in [5], [7]. In the first, the behaviour of $F_{\Phi_{v}}^{*}, F_{\Phi_{v}}$ and a certain zeta function $Z\left(\omega, \Phi_{v}\right)=\int_{X_{v}} \omega(f(x)) \Phi_{v}(x)|d x|_{v}$, for $\omega$ a quasi-character of $k_{v}^{x}$, are related. The asymptotic behaviour of $F_{\Phi_{v}}, F_{\Phi_{v}}^{*}$ is intimately related to the meromorphic continuation of $Z\left(\omega, \Phi_{v}\right)$, the information coming from the Hironaka resolution of $f=0$. (iii) is studied in the second work, and it should be thought of as providing the answer to the question: when can the measure $\left|\theta_{0}\right|_{v}$ on the open subset $U(0)_{v} \subset f^{-1}(0)_{v}$ be extended to a measure on $f^{-1}(0)_{v}$ ?

The importance of the theorem is that, depending solely on the geometric nature of the singularities of $f=0$ and given that the estimates of (ii) hold, the measures $d \mu_{g}$ of the abstract Poisson Formula are explicitly given by the $\left|\theta_{i}\right|_{v}$. The condition (P) of (iii) is too technical in nature to even state here. The interested reader is urged to read [7].

(2) Global CASE. The polynomial $f$ also gives rise to a continuous mapping, $f: X_{A} \rightarrow k_{A}$. Set

$$
F_{\Phi}^{*}\left(i^{*}\right)=\int_{X_{A}} \Phi(x) \psi\left(i^{*} f(x)\right)|d x|_{A},
$$

which defines a bounded, continuous function on $k_{A}$, for all $\Phi \in \mathcal{S}\left(X_{A}\right)$. In the global case we can describe a dense subspace of $\mathcal{S}\left(X_{A}\right)$. It is the C-space of functions of the form $\Pi_{v} \Phi_{v}$, where $\Phi_{v} \in \mathcal{S}\left(X_{v}\right)$ for every $v$, with $\Phi_{v}=$ the characteristic function of $X_{v}^{0}$, for almost all $v$. Now the measures $\left|\theta_{i}\right|_{v}(i \in k)$ exist for every $v$, but their restricted direct product $\left|\theta_{i}\right|_{A}$ may or may not exist on $U(i)_{A}$ and even when it does exist, the image measure under the mapping $U(i)_{A} \rightarrow X_{A}$ may not exist.

TheOREM 2 (IGUSA) [7]. Suppose (1) that $f$ is homogeneous of degree $m \geqslant 2$, over a global field $k$, with $\operatorname{chr}(k) \nmid m$ and that a Hironaka resolution exists for $f=0$. (2) $\operatorname{codim}\left(S_{f}\right.$ in $\left.f^{-1}(0)\right) \geqslant 2$. (3) For almost all $p$-fields $k_{v},\left|F_{v}^{*}\left(i^{*}\right)\right| \leqslant$ $\max \left(1,\left(i^{*}\right)_{v}\right)^{-\sigma}$ for a fixed $\sigma>2$, for every $i^{*} \in k_{v}$. Here $\sigma$ is to be independent of $v$ and $F_{v}^{*}=F_{\Phi_{v}}^{*}$ for $\Phi_{v}$ the characteristic function of $X_{v}^{0}$.

Then, for every $i \in k,\left|\theta_{i}\right|_{A}$ exists on $U(i)_{A}$ and its image measure exists on $X_{A}$. The sum of these image measures on $X_{A}$ is tempered; condition (B) holds for the mapping $X_{A} \stackrel{f}{\rightarrow} k_{A}$ and the discrete subgroup $k \subset k_{A}$. Further we have the Poisson Formula:

$$
\sum_{i \in k} \int_{U(i)_{A}} \Phi\left|\theta_{i}\right|_{A}=\sum_{i^{*} \in k} \int_{X_{A}} \Phi(x) \psi\left(i^{*} f(x)\right)|d x|_{A}
$$

This can be written as $\sum_{i \in k}\left|\theta_{i}\right|_{A}=\sum_{i^{*} \in k} \psi\left(i^{*} f(x)\right)$, as an identity of distributions.

REMARK. Condition (2) is easy to verify. It means that the hypersurface $f=0$ is irreducible and normal. The usefulness of this theorem is that it 
reduces a proof of the Poisson Formula to the verification of the estimate in (3), for almost all nonarchimedean valuations.

The proof is an assembling of the local data, careful estimates in the asymptotic expansions and Theorem 1.

Now this analysis has only incidentally seemed to be number theory-in so far as the objects constructed start from number fields, in some cases, but it has been much more harmonic analysis and algebraic geometry. Further, how can one ascertain that condition (3) of Theorem 2 is met? To indicate the number theory involved and to answer the above question, let us consider an important special case. This in itself will require another massive dose of algebraic geometry.

Suppose $S_{f}=\{0\}$, i.e. assume that the projective hypersurface defined by $f=0$ is nonsingular. Introduce coordinates in $X$ with respect to a $k$-basis for $X_{k}$. Then, by the Hilbert Nullstellensatz

$$
x_{i}^{\rho}=\sum_{j} A_{i j}(x) \frac{\partial f}{\partial x_{j}}(x), \quad 1 \leqslant i \leqslant n, \quad A_{i j} \in k\left[x_{1}, \ldots, x_{n}\right]
$$

for some $\rho$. We say a valuation $v$ or $k$ is "good" if

(i) $v \notin S_{\infty}$,

(ii) the coefficients of $f, A_{i j}$ belong to $\mathfrak{D}_{p_{v}}$,

(iii) $\psi_{v}$ is of conductor 1 i.e. $\psi_{v}=1$ on $\mathfrak{S}_{p_{v}}$ but $\psi_{v} \neq 1$ on $P_{v}^{-1}$. These conditions exclude only finitely many $v$ 's. For the rest we have

Lemma. For $v$ a "good" valuation, $\Phi_{v}=$ the characteristic function of $X_{v}^{0}$, $i^{*} \in k_{v}$ of order $-e \leqslant 0$, we have

$$
F_{\Phi_{v}}^{*}\left(i^{*}\right)=\left\{\begin{array}{l}
q^{n[-e / m]}, \quad e \neq 1(\bmod m), \\
q^{-n((e-1) / m+1)} \sum_{t\left(\bmod p_{v}\right)} \psi_{v}\left(\pi^{e-1} i^{*} t\right) N_{1}(t), \quad e \equiv 1(\bmod m)
\end{array}\right.
$$

where $q=q_{v}=\operatorname{card}\left(\mathfrak{D}_{v} / p_{v}\right) \cdot N_{1}(t)=\#\left\{a\left(\bmod p_{v}\right) \mid f(a) \equiv t\left(\bmod p_{v}\right)\right\}$ and $\pi$ is the uniforming parameter in $S_{v}=S_{p_{v}}$.

By the very definition $F_{\Phi_{v}}^{*}\left(i^{*}\right)=\int_{X_{0}^{0}} \psi_{v}\left(i^{*} f(x)\right)|d x|_{v}$ and now hidden behind the symbolism of integration, in this $p$-adic case, is the usual number theory of counting the number of solutions to polynomial equations, since basically such integrals can only be evaluated if we can use orthogonality of characters and one must count in how many ways a given situation arises.

For $e \neq 1(\bmod m)$, we see immediately that $\left|F_{v}^{*}\left(i^{*}\right)\right| \leqslant \max \left(1,\left|i^{*}\right|_{v}\right)^{-n / m}$ for such $i^{*} \in k_{v}$, but for $e \equiv 1(\bmod m)$, we must estimate $N_{1}(t)$. For $n=2$, Gauss sums yield the required estimates, but for $n \geqslant 3$, we consider the projective hypersurface $\bar{f}(x)-t x_{0}^{m}$, defined over the finite field $F_{q}$. Here, reduction of $f \bmod p_{v}$ makes sense, since $v$ is assumed "good", and these give rise to absolutely irreducible, nonsingular varieties. To estimate $N_{1}(t)$ we use Deligne's theorem on the Weil conjectures [4]. Deligne's theorem asserts: there exist complex numbers $\alpha_{1}, \alpha_{2}, \ldots$ of absolute value $q^{n / 2-1}$ such that $N_{1}(0)=q^{n-1}+(-1)^{n}(q-1) \Sigma_{i} \alpha_{i}$; and complex numbers $\alpha_{1}(t), \alpha_{2}(t), \ldots$ of 
absolute value $q^{n / 2-1}$, depending on $t \neq 0$, such that

$$
N_{1}(t)=q^{n-1}-(-1)^{n}\left(\sum_{i} \alpha_{i}+\sum_{j} \alpha_{j}(t)\right)
$$

where the number of $\alpha_{i}$ 's, $\alpha_{j}(t)$ 's depends only on $n, m$. Thus again we can estimate $\left|F_{v}^{*}\left(i^{*}\right)\right| \leqslant \max \left(1,\left|i^{*}\right|_{v}\right)^{-n / m}$.

Whence, if $n>2 m$, the Poisson Formula in Theorem 2 is valid for a form $f$ of degree $m \geqslant 2$, if $f=0$ is an irreducible, normal variety.

This example should indicate why the analysis is really number theory, as well as the depth of Igusa's results.

4. An application of the Poisson Formula. Keeping all the assumptions on $f$, namely that it be a homogeneous form of degree $m, \operatorname{chr}(k) \nmid m, S_{f}=(0)$ with $n>2 m$, we define two distributions

$$
E(\Phi)=\Phi(0)+\sum_{i^{*} \in k} \int_{X_{A}} \psi\left(i^{*} f(x)\right) \Phi(x)|d x|_{A},
$$

and $E^{\prime}(\Phi)=\sum_{\xi \in X_{k}} \Phi(\xi)$. Note that $E^{\prime}$ is independent of $f$ and it exists for every $n$. The remarkable fact is that there is a relationship between these two distributions! For quadratic forms, Weil introduces the metaplectic group and proves a uniqueness result on distributions that have certain invariance properties under this group. For higher degree forms, no such general group is known, but Igusa introduces a smaller one, which still enables him to establish a relationship. This group is modelled after the Weil metaplectic group. Set $G_{A}=k_{A} \times k_{A}^{x}$ and define $(u, t) \cdot\left(u^{\prime}, t^{\prime}\right)=\left(u+t^{m} u^{\prime}, t t^{\prime}\right)$, which makes $G_{A}$ into a locally compact group. Define an action of $G_{A}$ on $\mathcal{S}\left(X_{A}\right)$ by: for $g=(u, t),(U(g) \Phi)(x)=|t|_{A}^{n / 2} \psi(u f(x)) \Phi(t x)$. For simplicity, introduce the notation: if $\phi, \phi^{\prime}$ are complex valued functions on a set $X$, write $\phi(x) \prec \phi^{\prime}(x)$ if there is some constant $c$ such that $|\phi(x)| \leqslant c \phi^{\prime}(x)$ for all $x \in X$. Also if $g=(u, t)$, write $|g|_{A}=|t|_{A}^{m}$. Then

TheOREM 3 (Igusa [8]). (i) the function " $g \rightarrow\left(E^{\prime}-E\right)(U(g) \Phi)$ " is a $G_{k}$-invariant function on $G_{A}$, for every $\Phi \in \mathcal{S}\left(X_{A}\right)$.

(ii) if $n \gg m$, then for any given $\Phi \in \mathcal{S}\left(X_{A}\right)$, there exists $\epsilon>0$ such that

$$
\int_{k_{A} / k}\left(\left(E^{\prime}-E\right) U(g) \Phi\right)|d u|_{A} \prec|g|_{A}^{1+\epsilon-n / 2 m}
$$

on the subset of $G_{A}$ defined by $|g|_{A} \leqslant 1$.

This is a truly remarkable result, since as we have remarked, the $E^{\prime}$ does not depend on $f$. Actually more is true. The function defined in (i) vanishes to an order $(n / 2 m)-1$ at the $k$-rational "boundary points of $G_{A}$ ", a result analogous to the classical theorem which asserts that the theta series defined by a quadratic form behaves, up to lower order terms, like an Eisenstein series at every cusp. The proof of Igusa's Theorem 3 heavily uses the two expressions for $E(\Phi)$ given by the Poisson Formula of Theorem 2.

We are now ready to derive certain cases of the Davenport-Birch theorem as a corollary of the foregoing analysis and we give the details to show how it all fits together. 
Corollary. Keeping all the previous assumptions on $f$, if $n \gg m$ then the nonsingular hypersurface defined by $f=0$ has a $k$-rational point if and only if it has a $k_{v}$-rational point for every $v$.

Proof. For $t(u)=U(u, 1), d(t)=U(0, t)$, we have $U(g)=U(u, t)=$ $t(u) d(t)$, while $(t(u) \Phi)(x)=\psi(u f(x)) \Phi(x),(d(t) \Phi)(x)=|t|_{A}^{n / 2} \Phi(t x)$. So

$$
\left(E^{\prime}-E\right)(t(u) \Phi)=\sum_{\xi \in X_{k}}(t(u) \Phi)(\xi)-\sum_{i \in k} \int_{U(i)_{A}}(t(u) \Phi)\left|\theta_{i}\right|_{A}
$$

by the Poisson Formula. Now in the sum over $X_{k}$, group together the terms such that $f(\xi)=i$, so then $\left(\left(E^{\prime}-E\right) t(u) \Phi\right)=\sum_{i \in k} c_{i}(\Phi) \psi(i u)$, where $c_{i}(\Phi)=$ $\sum_{\xi \in U(i)_{k}} \Phi(\xi)-\int_{U(i)_{A}} \Phi\left|\theta_{i}\right|_{A}$, for every $i \in k$. By Theorem 3(i), the function " $u \rightarrow\left(\left(E^{\prime}-E\right) t(u) \Phi\right)$ " is a $k$-invariant function on $k_{A}$, whence calculating its Fourier coefficients we have

$$
c_{i}(\Phi)=\int_{k_{A} / k}\left(\left(E^{\prime}-E\right) t(u) \Phi\right) \psi(-i u)|d u|_{A} .
$$

In particular, for $i=0$ and $\Phi$ replaced by $d(t) \Phi$, we have

$$
c_{0}(d(t) \Phi)=\int_{k_{A} / k}\left(\left(E^{\prime}-E\right) t(u) d(t) \Phi\right)|d u|_{A} \prec|t|_{A}^{m(1+\epsilon-n / 2 m)}
$$

for some $\epsilon>0$, on the subset of $k_{A}^{x}$ defined by $|t|_{A} \leqslant 1$, by Theorem 3(ii).

Here $\epsilon$ depends on the choice of $\Phi$. But the original expression for $c_{0}$ now gives:

$$
\begin{aligned}
c_{0}(d(t) \Phi) & =\sum_{\xi \in U(0)_{k}}(d(t) \Phi)(\xi)-\int_{U(0)_{A}}(d(t) \Phi)\left|\theta_{0}\right|_{A} \\
& =|t|_{A}^{n / 2}\left(\sum_{\xi \in U(0)_{k}} \Phi(t \xi)-\int_{U(0)_{A}} \Phi(t x)\left|\theta_{0}(x)\right|_{A}\right) .
\end{aligned}
$$

Change variables in the integral over $U(0)_{A}, x \rightarrow t^{-1} x$; the explicit nature of the measure $\theta_{0}$ now gives us $\left|\theta_{0}\left(t^{-1} x\right)\right|_{A}=|t|_{A}^{m-n}\left|\theta_{0}(x)\right|_{A}$. This explains why we were so concerned about finding the explicit nature of the $d \mu_{g}$ in the abstract Poisson Formula! Making the appropriate substitutions, this yields

$$
\sum_{\xi \in U(0)_{k}} \Phi(t \xi)=|t|_{A}^{m-n}\left(\int_{U(0)_{A}} \Phi\left|\theta_{0}\right|_{A}+0\left(|t|_{A}^{m \epsilon}\right)\right) \text { as }|t|_{A} \rightarrow 0 .
$$

But the support of $\left|\theta_{0}\right|_{A}$ is $U(0)_{A}$ and so choosing $\Phi$ to be nonnegative, not identically zero on $U(0)_{A}$, we have $\int_{U(0)} \Phi\left|\theta_{0}\right|_{A}>0$ if $U(0)_{\mathrm{A}} \neq \varnothing$. But now $n>2 m$ at least so that the right-hand side of (2) is $>0$, for small $|t|_{A}$, whence we must have the left-hand side $>0$, so in particular $U(0)_{k} \neq \varnothing$. That is, we have $U(0)_{A} \neq \varnothing$ implies $U(0)_{k} \neq \varnothing$ as required.

The above result is an excellent example of the blending of analysis and algebraic geometry that has come about from the Siegel Formula, to achieve results of a number theoretical nature; Igusa's work having extended the earlier quadratic forms results of Weil. The above corollary is, in spirit, how Weil proves the Hasse-Minkowski theorem, but it is only a qualitative consequence of the Siegel Formula, which has also quantitative aspects [16]. 
5. Weil's formulation of the Siegel Formula [16]. Having seen some of the developments resulting from the Siegel Formula, let us now look at a special case of Weil's theory.

Let $k$ be a number field, $f$ a nondegenerate quadratic form on $k^{m}$ defined by $f(u)=^{t} u h u$, for $h \in M_{m}(k)$. Let $X_{k}=M_{m, n}(k)$, the space of all linear mappings $k^{n} \rightarrow k^{m}$ and for $x \in X_{k}, f \circ x$ is a quadratic form on $k^{n}$, with matrix ${ }^{t} x h x$. Let $I(X)_{k}$ denote the space of all quadratic forms on $k^{n}$. We regard $X_{k}, I(X)_{k}$ as the $k$-rational points of varieties $X, I(X)$ defined over $k$ and define $i_{X}: X \rightarrow I(X)$ by $i_{X}(x)={ }^{t} x h x$. Let $G=$ the group of matrices in $M_{m}$ which keep the form $f$ invariant and for each $i \in I(X)$, set $U(i)=\{x \in$ $X \mid i_{X}(x)=i$, rank $x=n$ \}. Then $U(i)$ is a $G$-orbit. In [16], Weil considers the more general situation where $X$ is a module over a semisimple algebra and then $G$ is one of the classical groups, but to illustrate the ideas, we discuss only the orthogonal group, which in the next section, we relate to Siegel's work. Further, let $S p=S p(X)$ denote the algebraic group of matrices $s \in$ $M_{2 n}$ satisfying ${ }^{t}$ ses $=e$, where $e=\left(\begin{array}{cc}0 & 1 \\ -1_{n} & 0\end{array}\right)$. The group of matrices $\left\{p=\left(\begin{array}{ll}a & b \\ 0 & d\end{array}\right)\right.$ $\in S p\}$ forms a parabolic subgroup of $S p$. Now each of the objects $X, G, S p, P$ are algebraic varieties defined over $k$ so we can attach to them their adelisations $X_{A}, G_{A}, S p_{A}, P_{A}$, and notice that $X_{A}$ is a locally compact abelian group. There exists a unitary representation $\mathfrak{r}$ of $\operatorname{Sp}(X)_{k}$ on $L^{2}\left(X_{A}\right)$ defined by:

$$
\begin{aligned}
& \mathfrak{r}\left(\left(\begin{array}{cc}
\alpha & 0 \\
0 & \alpha^{-1}
\end{array}\right) \Phi\right)(x)=\Phi(x \alpha) \quad \text { if } \alpha \in M_{n}(k), \text { invertible. } \\
& \mathfrak{r}\left(\left(\begin{array}{cc}
0 & \gamma \\
-\gamma^{t}-1 & 0
\end{array}\right) \Phi\right)(x)=\hat{\Phi}(x \gamma) \quad \text { for } \gamma \in M_{n}(k), \text { invertible. } \\
& \mathfrak{r}\left(\left(\begin{array}{ll}
I & \rho \\
0 & 1
\end{array}\right) \Phi\right)(x)=\Phi(x) \chi\left(\frac{1}{2} \operatorname{tr}\left({ }^{t} x x \rho\right)\right) \quad \text { for } \rho={ }^{t} \rho \in M_{n}(k) .
\end{aligned}
$$

Here $\hat{\Phi}$ is the Fourier transform of $\Phi$ defined by

$$
\hat{\Phi}(y)=\int_{X_{A}} \Phi(x y) \chi\left(\operatorname{tr}\left({ }^{t} x h y\right)\right)|d x|_{A}, \quad\left(y \in X_{A}\right)
$$

and $\chi$ is a character on $A=A_{k}$ such that $(x, y) \rightarrow \chi(x y)$ puts $A$ into duality with itself in such a way that $k=k^{\perp}$. The space $\delta\left(X_{A}\right)$ is mapped to itself by the $r(s), s \in \delta p(X)_{k}$. The Eisenstein-Siegel series is now defined by

$$
E(\Phi)=\sum_{P_{k} \backslash S p_{k}}(\mathrm{r}(s) \Phi)(0) .
$$

This is absolutely convergent for all $\Phi \in \mathcal{S}\left(X_{A}\right)$, uniformly on every compact subset of $\mathcal{S}\left(X_{A}\right)$, provided $m>2 n+2$.

In fact, $E(\Phi)=\sum_{Y} E_{Y}(\Phi)$ where the summation is extended over all subspaces $Y \subset X_{k}$ which satisfy $M_{m}(k) Y=Y$. In particular,

$$
E_{X}(\Phi)=\sum_{\rho={ }^{\prime} \rho \in M_{n}(k)} \int_{X_{A}} \Phi(x) \chi\left(\operatorname{tr}\left({ }^{t} x h x \rho\right)\right)|d x|_{A}
$$

for a suitable choice of coset representatives. The abstract Poisson Formula 
gives the Fourier transform of the function

$$
\rho \rightarrow \int_{X_{A}} \Phi(x) \chi\left(\operatorname{tr}\left({ }^{t} x h x \rho\right)\right)|d x|_{A}
$$

as the integral $\int_{U(i)_{A}} \Phi\left|\theta_{i}\right|_{A}$, if $i \in I(X)_{k}$.

$E$ is a tempered positive measure, with an invariance property under $S p_{k}$ and $G_{A}$, “ $\Phi(x) \rightarrow \Phi(g x)$ ".

Consider now another distribution, an integral of a theta series given by $I(\Phi)=\int_{G_{A} / G_{k}} \sum_{\xi \in X_{k}} \Phi(g \xi) d \nu(g)$ where $\nu$ is the Haar measure on $G_{A}$, normalized to make $G_{A} / G_{k}$ have volume $1 . I(\Phi)$ is absolutely convergent for all $\Phi \in \mathcal{S}\left(X_{A}\right)$ if $r=0$ or $m-r>n+1$, where $r=$ the index of $f$.

The Siegel Formula is now the equality $I=E$ if $m>2 n+2$. Restricting the measures $I, E$ to $U(i)_{A}$, one has

$$
\int_{G_{A} / G_{k}} \sum_{\xi \in U(i)_{k}} \Phi(g \xi) d \nu(g)=\int_{U(i)_{A}} \Phi\left|\theta_{i}\right|_{A} \quad \text { for } i \in I(X)_{k} .
$$

We mention this result, since the Hasse principle is a simple corollary: $U(i)_{A} \neq \varnothing$ implies that $U(i)_{k} \neq \varnothing$. We saw a similar application made for forms of degree more than two.

6. Deriving Siegel's result from Weil's theorem, $k=\mathbf{Q}$. There are two distinct cases, depending on whether $r=0$ (the definite case) or $r>0$ (indefinite case), requiring different treatment. For brevity we shall treat only the definite case, even though this necessitates the complication of genus, class considerations. For $k$ a number field, $h={ }^{t} h \in M_{m}(k), \operatorname{det}(h) \neq 0$, set $G=\left\{\left.u \in M_{m}(k)\right|^{t} u h u=h\right\}$, a reductive group, defined over $k$. Write $\mathcal{S}=\{a$ $\in M_{m}(k) \mid a={ }^{t} a$, $\left.\operatorname{det}(a) \neq 0\right\}$ and $\tilde{S} \tilde{L}_{m}=\left\{a \in M_{m}(k) \mid(\operatorname{det} a)^{2}=1\right\}$. We say $h, h^{\prime} \in \mathcal{S}$ belong to the same genus if $h^{\prime}=h[g]={ }^{t} g h g$ for some $g \in\left(\tilde{S} \tilde{L}_{\tilde{p}}\right)_{\mathcal{S}_{\infty}}$ and we say $h, h^{\prime} \in \mathcal{S}$ belong to the same class if $h^{\prime}=h[\gamma]$ for $\gamma \in \tilde{S} L(\sigma)$, where $\sigma=$ the ring of integers of $k$. Since $\tilde{S} \tilde{L}_{m}(\sigma) \subset\left(\tilde{S} \tilde{L}_{m}\right)_{S_{\infty}}$, each class belongs to a genus. In fact we have the following

Propositrion. Every genus consists of a finite number of classes. Moreover if $G_{A}=\amalg_{a} G_{S_{\infty}} a G_{k}$ is the double coset decomposition, then this corresponds to the class decomposition of the genus of $h$ (recall $G$ was defined in terms of $h$ ) and the correspondence is explicitly given by: $G \subset \tilde{S} \tilde{L}_{m}$ and $\left(\tilde{S} \tilde{L}_{m}\right)_{A}=\left(\tilde{S} \tilde{L}_{m}\right)_{S_{\infty}}$. $\left(S L_{m}\right)_{k} \ni a=g \cdot \gamma^{-1}$. Then $G_{S_{\infty}} a G_{k} \rightarrow$ class of $h[g]$, where $a=g \gamma^{-1}, g \stackrel{\infty}{\in}$ $\left(\tilde{S} \tilde{L}_{m}\right)_{S_{\infty}}, \gamma \in\left(S L_{m}\right)_{k}$.

Restrict attention now to $k=Q, \mathrm{~h}>0$, positive definite form. Then $G_{S_{\infty}}=G_{\mathbf{R}} \cdot \Pi_{p \neq \infty} G_{p}^{\circ}$ is a compact group, whence $G_{A} / G_{Q}=\amalg_{i} G_{S_{\infty}} a_{i} G_{Q} / G_{Q}$ is compact, $\cong \amalg_{i} G_{S_{\infty}} /\left(G_{S_{\infty}} \cap a_{i} G_{Q} a_{i}^{-1}\right)$ and write $e_{i}=\operatorname{card}\left(G_{S_{\infty}} \cap\right.$ $\left.a_{i} k G_{Q} a_{i}^{-1}\right)$. Let us now examine the integral $I(\Phi)$ introduced by Weil. This is convergent for all $m, n$ and $\nu\left(G_{A} / G_{Q}\right)=1$ gives $\nu\left(G_{S_{\infty}}\right)=\left(\Sigma_{i} 1 / e_{i}\right)^{-1}$. Thus

$$
\begin{aligned}
I(\Phi) & =\int_{G_{A} / G_{Q}}\left(\sum_{\xi \in X_{Q}} \Phi(u \xi)\right) d \nu(u) \\
& =\sum_{i} e_{i}^{-1} \int_{G_{S \infty}}\left(\int_{\xi \in X_{Q}} \Phi\left(u a_{i} \xi\right)\right) d \nu(u) .
\end{aligned}
$$


Take $\Phi=\Phi_{0} \otimes \Phi_{\infty}$ where $\Phi_{0}=$ the characteristic function of $\Pi_{p} X_{p}^{0}$ and $\Phi_{\infty}\left(x_{\infty}\right)=e\left(\frac{1}{2}\left(\operatorname{tr} h\left[x_{\infty}\right] \tau\right)\right)$ for $\tau \in \mathcal{S}_{n}$, the Siegel upper half space of complex matrices $\{X+i Y \mid X, Y$ symmetric real, $Y>0\}$ and $e(--)=\mathrm{e}^{2 \pi \mathrm{i}--}$. Then $\Phi\left(u a_{i} \xi\right) \neq 0$ if and only if the finite part of $u a_{i} \xi$ is integral, i.e. $u a_{i} \xi \in X_{S_{\infty}}$. But writing $a_{i}=g_{i} \gamma_{i}^{-1}$ for $g_{i} \in\left(\tilde{S} \tilde{L}_{m}\right)_{S_{\infty}}, \gamma_{i} \in\left(S L_{m}\right)_{Q}$ then $u, g_{i}$ keep $X_{S_{\infty}}$ stable, so $u a_{i} \xi \in X_{S_{\infty}}$ iff $\gamma_{i}^{-1} \xi \in X_{S_{\infty}} \cap X_{Q}=X_{\mathbf{z}}$. Hence

$$
\begin{aligned}
(1) \cdots \int_{G_{S_{\infty}}} \sum_{\xi \in X_{Q}} \Phi\left(u a_{i} \xi\right) d \nu(u) & =\int_{G_{S_{\infty}}}\left(\sum_{\xi \in X_{\mathbf{Z}}} \Phi\left(u g_{i} \xi\right)\right) d \nu(u) \\
& =\int_{G_{S_{\infty}}} \sum_{\xi \in X_{\mathbf{Z}}} e\left(\frac{1}{2} \operatorname{tr} h\left[u_{\infty}\left(g_{i}\right)_{\infty} \xi\right] \tau\right) d \nu(u) .
\end{aligned}
$$

But, writing $h_{i}=h\left[\gamma_{i}\right]$, we see that

$$
h\left[u_{\infty}\left(g_{i}\right)_{\infty} \xi\right]=h\left[\left(g_{i}\right)_{\infty} \xi\right]=h_{i}[\xi]
$$

so

$$
\text { (1) . . } \int_{G_{S_{\infty}}} \sum_{\xi \in X_{\mathbf{Z}}} e\left(\frac{1}{2} \operatorname{tr} h_{i}[\xi] \tau\right) d \nu(u)=\nu\left(G_{S_{\infty}}\right) \sum_{\xi \in X_{\mathbf{Z}}} e\left(\frac{1}{2} \operatorname{tr} h_{i}[\xi] \tau\right)
$$

since now the integral is independent of $u$. But Siegel calls $\sum_{\xi \in X_{\mathbf{z}}} e\left(\frac{1}{2} \operatorname{tr} h_{i}[\xi] \tau\right)$ $=f_{i}(\tau)$, the analytic invariant of the class of $h_{i}$, so we have

$$
I(\Phi)=\frac{\sum_{i} f_{i}(\tau) / e_{i}}{\sum_{i} 1 / e_{i}}=F(\tau)
$$

the analytic invariant of the genus of $h$. The arithmetic meaning of these invariants is that in the sum $f_{i}(\tau)=\sum_{\xi \in X_{\mathbf{z}}} e\left(\frac{1}{2} \operatorname{tr}\left(h_{i}[\xi] \tau\right)\right)$, if we group together the terms $\xi$ for which $h_{i}[\xi]=\eta$, then if this occurs, say $A\left(h_{i}, \eta\right)$ times, we have $f_{i}(\tau)=\sum_{\eta \in X_{\mathbf{z}}} A\left(h_{i}, \eta\right) e^{\pi i \operatorname{tr}(\eta \tau)}$, the generating function for the number of representations of the $\eta$ by $h_{i}$. Then

$$
F(\tau)=\frac{\sum_{i} f_{i}(\tau) / e_{i}}{\sum 1 / e_{i}}=\sum_{\eta \in X_{\mathbf{Z}}} \bar{A}(h, \eta) e^{\pi i \operatorname{tr}(\eta \tau)}
$$

where $\bar{A}(h, \eta)=$ the mean of the number of representations of $\eta$ by the genus of $h$ (c f. Siegel [12]).

To explicate the other side of Weil's theorem, we need a convenient set of coset representatives for $P_{k} \backslash(S p)_{k}$. Let $\Theta=M_{m}$, with $x^{\imath}=h^{-1 t} x h, X=$ $M_{m, n}$, a left $\mathbb{Q}$-module. The set $Q(X / \mathbb{Q})$ of all sesquilinear, hermitian mappings $X \times X \rightarrow Q$ can be identified with $\left\{\rho \in M_{n} \mid \rho={ }^{t} \rho\right\}$ by $(x, y) \rightarrow$ $x \rho^{t}(h y)$, with $q(x)=\frac{1}{2} \operatorname{tr}\left(x \rho^{t}(h x)\right)=\frac{1}{2} \operatorname{tr}(h[x] \rho)$ the associated quadratic form. Then, to obtain coset representatives, we take $X_{1} \subset X$, and $\mathscr{Q}$-submodule and choose a complement as follows: if rank $\left(X_{1}\right)=r(0 \leqslant r \leqslant n)$, since $X=I_{1}$ $\oplus \cdots \oplus I_{n}$ of minimal left ideals, $X_{1}$ is one of $\left(\begin{array}{c}n \\ r\end{array}\right)$ possible submodules of rank $r$. Fix $v \in S L_{n}(\mathbf{Z})$ to put them in the order $I_{1} \oplus \cdots \oplus I_{r}$, i.e. $X_{1} v=$ $M_{m, r} \oplus 0$ and choose $X_{2}=\left(0 \oplus M_{m, n-r}\right) v^{-1}$. Pick $\rho_{1} \in Q\left(X_{1} / \Theta\right)$, then the collection $\left\{\left(X_{1}, \rho_{1}\right)\right\}$ forms a complete set of representatives, with $q_{1}\left((\omega, 0) v^{-1}\right)=\frac{1}{2} \operatorname{tr}\left(h[\omega] \rho_{1}\right)$, for $\rho_{1}={ }^{t} \rho_{1} \in M_{r}, \omega \in M_{m, r}$ and then $\left(r\left(s_{0}\right) \Phi\right)(0)$ $=\int_{(M m, r) A} \Phi\left((\omega, 0) v^{-1}\right) \epsilon\left(\frac{1}{2} \operatorname{tr} h[\omega] \rho_{1}\right)|d \omega|_{A}$. Here $\epsilon=\prod_{p} \epsilon_{p}$ of the local func- 
tions where $\epsilon_{\infty}=e$ and $\epsilon_{p}(x)=e^{2 \pi i n / p^{\nu}}$ where $p^{\nu} x$ integral and $n \equiv p^{v} x$ $\left(\bmod p^{\nu}\right)$. So that

$$
E(\Phi)=\sum_{\left(X_{1}, \rho_{1}\right)} \int_{\left(M_{m, r}\right)_{A}} \Phi\left((\omega, 0) v^{-1}\right) \epsilon\left(\frac{1}{2} \operatorname{tr} h[\omega] \rho_{1}\right)|d \omega|_{A} .
$$

Thus, for the $\Phi=\Phi_{0} \otimes \Phi_{\infty}$ as before, this gives

$$
\begin{aligned}
E(\Phi)= & \sum_{\left(X_{1}, \rho_{1}\right)} \prod_{p} \int_{M_{m, r}\left(Q_{p}\right)} \Phi_{p}\left(\left(\omega_{p, 0}\right) v^{-1} \epsilon_{p}\left(\frac{1}{2} \operatorname{tr} h\left[\omega_{p}\right] \rho_{1}\right)\right)|d \omega|_{p} \\
& \times \int_{M_{m, r}(\mathbf{R})} \Phi_{\infty}\left(\left(\omega_{\infty}, 0\right) v^{-1}\right) \epsilon_{\infty}\left(\frac{1}{2} \operatorname{tr} h\left[\omega_{\infty}\right] \rho_{1}\right)|d \omega|_{\infty}
\end{aligned}
$$

Now to evaluate these.

(i) $\infty$-part. $\Phi_{\infty}\left(x_{\infty}\right)=e\left(\frac{1}{2} \operatorname{tr} h\left[x_{\infty}\right] \tau\right)$, while $h\left[\left(\omega_{\infty}, 0\right) v^{-1}\right]$ $={ }^{t} v^{-1} h\left[\left(\omega_{\infty}, 0\right)\right] v^{-1}$,so that $\operatorname{tr}\left(h\left[\left(\omega_{\infty}, 0\right) v^{-1}\right] \tau\right)=\operatorname{tr}\left(h\left[\omega_{\infty}, 0\right] v^{-1} \tau^{t} v^{-1}\right)$. Writing $v^{-1} \tau^{t} v^{-1}=\left(\begin{array}{cc}\tau_{1} & * \\ * & *\end{array}\right), h\left[\omega_{\infty}, 0\right]=\left(\begin{array}{c}h\left[\omega_{\infty}\right] \\ 0\end{array}\right)$ with $\tau_{1} \in \mathcal{S}_{r}$, the trace is $\operatorname{tr}\left(h\left[\omega_{\infty}\right] \tau_{1}\right)$. Thus

$$
\begin{aligned}
I_{\infty} & =\int_{M_{m, r}(\mathbf{R})} \Phi_{\infty}\left(\left(\omega_{\infty}, 0\right) v^{-1}\right) e_{\infty}\left(\frac{1}{2} \operatorname{tr} h\left[\omega_{\infty}\right] \rho_{1}\right)|d \omega|_{\infty} \\
& =\int_{M_{m, r}(\mathbf{R})} e\left(\frac{1}{2} \operatorname{tr} h\left[\omega_{\infty}\right]\left(\tau_{1}-\rho_{1}\right)\right)\left|d \omega_{\infty}\right|_{\infty}
\end{aligned}
$$

Lemma. For $S={ }^{t} S \in M_{m}(\mathbf{R}), S$ positive definite, $x \in M_{m, r}(\mathbf{R})$, define $\Phi_{S}(x)$ $=e^{-\pi \operatorname{tr}(x S x)}$; then its Fourier transform is given by

$$
\begin{aligned}
\left(\mathcal{G} \Phi_{S}\right)(x) & =\int_{M_{m, r}(\mathbf{R})} \Phi_{S}(y) e\left(\operatorname{tr}^{t} y x\right) d y \\
& =(\operatorname{det} S)^{-r / 2} \Phi_{S}^{-1}(x) .
\end{aligned}
$$

The proof is by contour integration. In particular, for $x=0$,

$$
\int_{M_{m, r}(\mathbf{R})} e^{-\pi \operatorname{tr}(x S x)} d x=(\operatorname{det} S)^{-r / 2} \text {. }
$$

Now

$$
I_{\infty}=\int_{M_{m, r}(\mathbf{R})} e^{\pi i\left(\operatorname{tr} h\left[\omega_{\infty}\right]\left(\tau_{1}-\rho_{1}\right)\right)}\left|d \omega_{\infty}\right|_{\infty}
$$

But $\tau_{1}-\rho_{1}=\left(X_{1}-\rho_{1}\right)+i Y_{1} \in \mathcal{S}_{r}, Y_{1}>0$, so

$$
I_{\infty}=\int_{M_{m, r}(\mathbf{R})} e^{-\pi \operatorname{tr}\left(h\left[\omega_{\infty}\right] Y_{1}\right)} e^{\pi i\left(\operatorname{tr} h\left[\omega_{\infty}\right]\left(X_{1}-\rho_{1}\right)\right)}\left|d \omega_{\infty}\right|_{\infty}
$$

and by analytic continuation, it suffices to evaluate this for $X_{1}=\rho_{1}$ i.e. $\tau_{1}-\rho_{1}$ purely imaginary. Since $Y_{1}>0$, we can write it $Y_{1}=Z^{t} Z, h={ }^{t} L L$, thus, by the above lemma,

$$
I_{\infty}=(\operatorname{det} h)^{-r / 2}\left(\operatorname{det}\left(\tau_{1}-\rho_{1}\right) i^{-1}\right)^{-m / 2} .
$$

(ii) p-part.

$$
I_{p}=\int_{M_{m, r}\left(\mathbf{Z}_{p}\right)} \epsilon_{p}\left(\frac{1}{2} \operatorname{tr} h\left[\omega_{p}\right] \rho_{1}\right)|d \omega|_{p}
$$


$\rho_{1}={ }^{t} \rho_{1} \in M_{r}(Q)$, so we can write it $\rho_{1}=\gamma_{1}^{-1} \delta_{1}$ for $\gamma_{1}, \delta_{1} \in M_{r}(\mathbf{Z})$, det $\gamma_{1}$ $>0$; for example if the denominator of $\rho_{1}=N$, set $\gamma_{1}=N I, \delta_{1}=N \rho_{1}$. We say the pair $\left(\gamma_{1}, \delta_{1}\right)$ has no left zero divisors if for $u \in M_{r}(\mathbf{Z}), \operatorname{det}(u) \neq 0$, the condition $u^{-1}\left(\gamma_{1}, \delta_{1}\right) \in M_{r, 2 r}(\mathbf{Z})$ implies $u \in \tilde{S} \tilde{L}_{r}(\mathbf{Z})$. Now, by the theorem on elementary divisors we may choose the $\left(\gamma_{1}, \delta_{1}\right)$ in the expression for $\rho_{1}$ to have no left zero divisors. We consider the additive subgroup $M_{m, r}\left(\mathbf{Z}_{p}\right) \gamma_{1} \subset$ $M_{m, r}\left(\mathbf{Z}_{p}\right)$ and noting that

$$
\begin{aligned}
\epsilon_{p}\left(\frac{1}{2} \operatorname{tr} h\left[\omega_{p}+\omega_{p}^{\prime} \gamma_{1}\right] \rho_{1}\right)= & \epsilon_{p}\left(\frac{1}{2} \operatorname{tr} h\left[\omega_{p}\right] \rho_{1}\right) \epsilon_{p}\left(\frac{1}{2} \operatorname{tr} h\left[\omega_{p}^{\prime} \gamma_{1}\right] \rho_{1}\right) \\
& \times \epsilon_{p}\left(\operatorname{tr}^{t} \omega_{p} h \omega_{p}^{\prime} \gamma_{1} \gamma_{1}^{-1} \delta_{1}\right) \\
= & \epsilon_{p}\left(\frac{1}{2} \operatorname{tr} h\left[\omega_{p}\right] \rho_{1}\right) \epsilon_{p}\left(\frac{1}{2} \operatorname{tr}^{t} \omega_{p}^{\prime} h \omega_{p}^{\prime} \delta_{1}{ }^{t} \gamma_{1}\right)
\end{aligned}
$$

since $h \in M_{m}(\mathbf{Z})$, we have

$$
\begin{aligned}
I_{p} & =\sum_{\omega_{p} \bmod } \sum_{M_{m, r}\left(\mathbf{z}_{p}\right) \gamma_{1}} \int_{M_{m, r}\left(\mathbf{z}_{p}\right) \gamma_{1}} \epsilon_{p}\left(\frac{1}{2} \operatorname{tr} h\left[\omega_{p}+\omega_{p}^{\prime} \gamma_{1}\right] \rho_{1}\right)\left(d \omega_{p}^{\prime} \gamma_{1}\right)_{p} \\
& =\sum_{\omega_{p} \bmod M_{m, r}\left(\mathbf{z}_{p}\right) \gamma_{1}} \epsilon_{p}\left(\frac{1}{2} \operatorname{tr} h\left[\omega_{p}\right] \rho_{1}\right) \int_{M_{m, r}\left(\mathbf{z}_{p}\right) \gamma_{1}} \epsilon_{p}\left(\frac{1}{2} \operatorname{tr} h[\omega] \rho_{1}\right)|d \omega|_{p}
\end{aligned}
$$

which, by the change of variable $\omega_{p}^{\prime}=\omega \gamma_{1}^{-1}$ becomes

$I_{p}=\sum_{\omega_{p} \bmod } \sum_{M_{m, r}\left(\mathbf{Z}_{p}\right) \gamma_{1}}\left|\operatorname{det} \gamma_{1}\right|_{p}^{m} \epsilon_{p}\left(\frac{1}{2} \operatorname{tr} h\left[\omega_{p}\right] \rho_{1}\right) \int_{M_{m, r}\left(\mathbf{Z}_{p}\right)} \epsilon_{p}\left(\frac{1}{2} \operatorname{tr} h\left[\omega_{p}^{\prime}\right] \gamma_{1}^{t} \delta_{1}\right)\left|d \omega_{p}^{\prime}\right|_{p}$

and denoting the integral $\delta_{p}$, its value being 1 or 0 as the character " $\omega_{p}^{\prime} \rightarrow$ $\epsilon_{p}\left(\frac{1}{2} \operatorname{tr} h\left[\omega_{p}^{\prime}\right] \gamma_{1}{ }^{t} \delta_{1}\right)$ " is trivial or not on the compact group $M_{m, r}\left(\mathbf{Z}_{p}\right)$, we have

$$
I_{p}=\left|\operatorname{det} \gamma_{1}\right|_{p}^{m} \sum_{\omega_{p} \bmod M_{m, r}\left(\mathbf{Z}_{p}\right) \gamma_{1}} \epsilon_{p}\left(\frac{1}{2} \operatorname{tr} h\left[\omega_{p}\right] \rho_{1}\right) \delta_{p} .
$$

Now $\gamma_{1} \in M_{r}(\mathbf{Z})$, so it is a $p$-adic unit for almost all $p$. For such $p$, we may take $\omega_{p}=0, \epsilon_{p}(0)=1$ so that $I_{p}=\left|\operatorname{det} \gamma_{1}\right|_{p}^{m} \delta_{p}$.

For the remaining $p, \gamma_{1}$ defines an injective mapping $T_{p}: \mathbf{Z}_{p}^{m r} \rightarrow \mathbf{Z}_{p}^{m r}$, and by elementary divisors again we see the index $\left[\mathbf{Z}_{p}^{m n}: T_{p}\left(\mathbf{Z}_{p}^{m n}\right)\right]=\left|\operatorname{det} T_{p}\right|_{p}$.

Thus, the $T: \mathbf{Z}^{m n} \rightarrow \mathbf{Z}^{m n}$ that $\gamma_{1}$ defines gives the isomorphism $\mathbf{Z}^{m n} / T\left(\mathbf{Z}^{m n}\right) \stackrel{\sim}{\rightarrow} \Pi_{p} \mathbf{Z}_{p}^{m n} / T_{p}\left(\mathbf{Z}_{p}^{m n}\right)$. Thus, assembling the parts $I_{\infty}, I_{p}$ for all $p$, we see

$$
\begin{aligned}
\left(\mathfrak{r}\left(s_{0}\right) \Phi\right)(0)= & \prod_{p}\left|\operatorname{det} \gamma_{1}\right|_{p}^{m} \sum_{\omega_{p} \bmod M_{m, r}\left(\mathbf{z}_{p}\right) \gamma_{1}} \epsilon_{p}\left(\frac{1}{2} \operatorname{tr} h\left[\omega_{p}\right] \rho_{1}\right) \delta_{p} \cdot I_{\infty} \\
= & \left|\operatorname{det} \gamma_{1}\right|^{-m}\left(\sum_{\omega \bmod M_{m, r}(\mathbf{Z}) \gamma_{1}} e\left(\frac{1}{2} \operatorname{tr} h[\omega] \rho_{1}\right)\right) \prod_{p} \delta_{p} \cdot(\operatorname{det} h)^{-r / 2} \\
& \cdot\left(\operatorname{det}\left(\tau_{1}-\rho_{1}\right) i^{-1}\right)^{-m / 2} \\
= & i^{m r / 2}(\operatorname{det} h)^{-r / 2}\left|\operatorname{det} \gamma_{1}\right|^{-m / 2}\left(\operatorname{det}\left(\gamma_{1} \tau_{1}-\delta_{1}\right)\right)^{-m / 2} \\
& \cdot \prod_{p} \delta_{p}\left(\sum_{\omega \bmod \gamma_{1}} e\left(\frac{1}{2} \operatorname{tr} h[\omega] \gamma_{1}^{-1} \delta_{1}\right)\right)
\end{aligned}
$$

whence $E(\Phi)=$ the sum of such terms over the coset representatives $\left(X_{1}, \rho_{1}\right)$. 
Siegel's main theorem then asserts that $E(\Phi)=F(\tau)$, giving another expression for the analytic invariant of the genus of $h$.

7. Other applications of these developments. The theory of the asymptotic expansions of $F_{\Phi}^{*}, F_{\Phi}$ developed by Igusa to establish the Poisson Formula, has another interesting application, settling a conjecture raised by BorevichShafarevich [2, p. 47]. Let $K$ be a $p$-adic field of characteristic zero, with $R$ the maximal compact subring in $K$ and $P$ the unique maximal ideal of $R$. Let $F_{q}=R / P$ and $f(x) \in R\left[x_{1}, \ldots, x_{n}\right]$ be any polynomial. Set $N(e)=$ $\#\left\{\xi \in R^{n}\left(\bmod p^{e}\right) \mid f(\xi) \equiv 0\left(\bmod p^{e}\right)\right\}$, and form the Poincare series $P(z)$ $=\sum_{e=0}^{\infty} N(e)\left(z / q^{n}\right)^{e}$.

\section{THEOREM 4 (IgUSA [9]). $P(z)$ is a rational function of $z$.}

We emphasize that this holds for any polynomial $f$ ! The method of proof again relates this arithmetically defined power series to an integral, in this case to $Z(s)=\int_{R^{n}}|f(x)|^{s} d x$, for $s$ a complex number with positive real part. An elementary computation, involving counting the number of solutions shows that $P\left(q^{-s}\right)=\left(1-q^{-s} Z(s)\right)\left(1-q^{-s}\right)^{-1}$. Thus it suffices to prove that the analytic continuation of $Z(s)$ is a rational function of $q^{-s}$. A Hironaka resolution for $f=0$ is used (which exists, since $\operatorname{chr} K=0$ is assumed) to study the analytic behaviour of $Z(s)$.

Classically one studied exponential sums of the type $\sum_{\xi \in \mathbf{Z}^{n} \cap \tau^{-1 J}} e\left(u_{\infty} f(\xi)\right)$, where $f \in \mathbf{Z}\left[x_{1}, \ldots, x_{n}\right], J$ an open set in $\mathbf{R}^{n}, \tau \in \mathbf{R}_{+}^{x}, u_{\infty} \in \mathbf{R}^{m}$ and $e(t)=$ $e^{2 \pi i t}$ as before. Such a sum behaves quite delicately as a function of $u_{\infty}, \tau$ [1]. For a suitable choice of function $\Phi$ and $g \in G_{A}$, this is just $E^{\prime}(U(g) \Phi)$ introduced by Igusa and so related to $E(U(g) \Phi)$ by his theory.

One final comment should be made. As is evident from the corollary to Theorem 3, a significant result can be obtained by using only such a small group $G$. Weil's metaplectic group is much larger, and gives also the complete Siegel Formula. Further, the use of the metaplectic group allows one to treat the situation where one has a mapping $X \stackrel{f}{\rightarrow} Y$ of affine varieties, with $\operatorname{dim} Y>1$; that is, of $f=\left(f_{1}, \ldots, f_{r}\right)$ of $r$ quadratic polynomials. The generalization of the metaplectic group for higher degree forms would be extremely useful, but is off in the future. It appears to be quite difficult, since the natural candidate that Igusa has introduced for a finite field [8] behaves in unexpected ways. For example $M p$ is not a central extension of $S L_{2}(K)$ and it turns out that every homomorphism $S L_{2}(K) \rightarrow M$ is in fact trivial. So there is much room for further developments.

\section{REFERENCES}

1. B. J. Birch, Forms in many variables, Proc. Roy. Soc. Ser. A 265 (1961 /62), 245-263.

2. Z. I. Borevich and I. R. Shafarevich, Number theory, Academic Press, New York, 1966.

3. H. Davenport, Cubic forms in sixteen variables, Proc. Roy. Soc. Ser. A 272 (1963), 285-303.

4. P. Deligne, Lectures in honor of W. V. D. Hodge, Cambridge, 1973.

5. J. I. Igusa, Complex powers and asymptotic expansions, I, J. Reine Angew Math. 268/269 (1974), 110-130; II, Ibid, 278/279 (1975), 307-321.

6. On a certain Poisson Formula, Nagoya Math. J. 53 (1974), 211-233.

7. Criteria for the validity of a certain Poisson Formula, Algebraic Number Theory, Kyoto, 1976, pp. 43-65. 
8. $223-244$.

9. ___ Some observations on higher degree characters, Amer. J. Math 99 (1977), 393-417.

10. M. Levin, A continuity problem in the Siegel-Weil formula, Tech. Report (1974), University of Maryland.

11. E. S. Selmer, The Diophantine equation $a x^{3}+b y^{3}+c z^{3}=0$, Acta Math. 85 (1951), 203-363.

12. C. L. Siegel, Lectures on the analytical theory of quadratic forms, Institute for Advanced Study Notes, 1934.

13. __ Lectures on quadratic forms, Tata Institute Notes, Bombay, India, 1957.

14. T. Tamagawa, Adeles, Proc. Sympos. Pure Math., vol 9, Amer. Math. Soc., Providence, R. I., 1966, pp. 113-121.

15. A. Weil, Sur certaines groupes d'operateurs unitaires, Acta Math. 111 (1964), 143-211.

16. __ Sur la Formule de Siegel dans la theorie des groupes classiques, Acta Math. 113 (1965), 1-87.

17. __ Adeles and algebraic groups, Lecture Notes, Institute for Advanced Study, Princeton, 1961.

Department of Mathematics, University of Washington, Seattle, Washington 98195 
